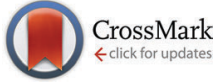

Cite this: Phys. Chem. Chem. Phys., 2016, 18, 27630

Received 20th June 2016, Accepted 2nd September 2016 DOI: $10.1039 / c 6 c p 04315 a$

www.rsc.org/pccp

\section{Mass effects in the photodissociation of homonuclear diatomic molecules in helium nanodroplets: inelastic collision and viscous flow energy exchange regimes}

\author{
Arnau Vilà and Miguel González*
}

\begin{abstract}
The influence of the mass in the photodissociation dynamics of a homonuclear diatomic molecule $\left(X_{2}\right)$, embedded in a superfluid helium nanodroplet $(T=0.37 \mathrm{~K}$ ) of 300 atoms, has been investigated using a hybrid quantum dynamics method recently proposed by us. Several hypothetical isotopic variants of $\mathrm{Cl}_{2}$ have been examined in order to make possible the analysis of a wide diversity of masses ( $m_{\mathrm{X}}$ : $\left.0.25 m_{\mathrm{Cl}}-1.50 m_{\mathrm{Cl}}\right)$. This is probably the first time that this problem has been considered theoretically. The photodissociation mechanism of $\mathrm{X}_{2}(\mathrm{~B})$ is very similar to that of $\mathrm{Cl}_{2}(\mathrm{~B})$ and the efficiency of the $\mathrm{X}_{2}$-helium energy exchange mechanism can be so great that it leads to the full and partial $(\approx 86 \%)$ geminate recombination for the lower masses explored $\left(m_{X}=0.25 m_{C l}\right.$ and $0.50 m_{C l}$, respectively). From the energy exchange perspective two dynamic regimes have been identified. The first regime occurs at the initial times of the photodissociation and corresponds to a perfectly inelastic collision (IC) between the atomic fragments $(X)$ and some helium atoms of the solvation shell. The second regime occurs when the atomic fragments are moving through the nanodroplet, which behaves as a viscous fluid (VF). The ICVF mechanism has probably general character in the photodissociation of molecules embedded in superfluid helium nanodroplets.
\end{abstract}

\section{Introduction}

Superfluid helium nanodroplets, $\left({ }^{4} \mathrm{He}\right)_{N}$, have attracted lots of interest from the scientific community, not only for exploring finite-size superfluidity but also because of their characteristics as a solvent. ${ }^{1,2}$ Their superfluidity, chemically inert character, ability to be doped with almost any chemical species, very low temperature $(0.37 \mathrm{~K})$ and large heat capacity make these systems ideal nanoreactors to investigate a wide variety of chemical processes. ${ }^{3,4}$ Another main feature of helium nanodroplets is their capacity to stabilize chemical species such as ions, molecules, nanoclusters ${ }^{5,6}$ and nanowires. ${ }^{7,8}$ This is important from a chemical perspective and it is of great interest to understand the impurity relaxation mechanism, i.e. how the energy is released from the dopant chemical species to the liquid helium.

The first experiments on this topic were focused on the cage effect in the fragmentation of photoionized molecules ${ }^{9-12}$ and rare-gas clusters ${ }^{13-16}$ embedded in helium nanodroplets. The energy relaxation by helium nanodroplets has also been studied experimentally considering neutral species (photoinduced

Departament de Química Física i IQTC, Universitat de Barcelona, c/ Martí i Franquès, 1, 08028 Barcelona, Spain. E-mail: miguel.gonzalez@ub.edu; Fax: +34934021231 isomerization of linear and bent isomers of $\mathrm{HCN}-\mathrm{HF}^{17}$ and photodissociation of alkyl iodides; R-I $+h \nu \rightarrow \mathrm{R}+\mathrm{I}) \cdot{ }^{18-20}$ In the former case the amount of population transfer has been found to be very different for pumping the two isomers; ${ }^{17}$ while in the second case the velocity and angular distributions of the photofragments were measured and an important cage effect, which strongly depends on the fragment mass, was observed. ${ }^{18-20}$ Most of the experiments pointed out that the cooling by helium is not a thermal evaporative process, i.e. the mean energy per evaporated helium atom is greater than the binding energy per atom in liquid helium $(\approx 7 \mathrm{~K})$. Furthermore, the cooling efficiency was found to depend on the nanodroplet size.

Different energy relaxation mechanisms have been proposed in the experimental works to understand the different features found. Thus, e.g., in the fragmentation of rare gas clusters the detection of $\mathrm{He}_{2}{ }^{+}$showed that the nanodroplet released the excess energy by the ejection of this ion, ${ }^{13}$ rather than through an evaporative process. Besides, the breakup of the nanodroplet was postulated to explain that the mean energy released per evaporated helium atom is greater for the smaller nanodroplets. ${ }^{11,12}$ Regarding the $\mathrm{R}-\mathrm{I}$ photodissociation, the results were interpreted in terms of hardsphere binary collisions between the fragments and the helium atoms. ${ }^{18}$ In this context it is clear that studying these kinds of processes theoretically is interesting, so as to obtain a deeper insight into the dopant species-helium energy exchange process. 
In a theoretical study of the $\mathrm{Cl}_{2}(\mathrm{~B})$ photodissociation in helium nanodroplets at $T=4 \mathrm{~K}$ (i.e. non-superfluid liquid helium) an important cage effect was determined, ${ }^{21}$ leading to a reduction of the $\mathrm{Cl}\left({ }^{2} \mathrm{P}_{3 / 2}\right)+\mathrm{Cl}^{*}\left({ }^{2} \mathrm{P}_{1 / 2}\right)$ fragment velocities compared with the gas phase result. A semiclassical path integral centroid molecular dynamics was used for liquid helium and a quantum wave packet dynamics for the impurities.

The fragmentation of rare gas clusters inside helium nanodroplets was studied theoretically employing a quantum/ classical strategy for the dopant, while the liquid helium was described by a friction force (acting only for atom velocities above the Landau critical velocity). ${ }^{22}$ This method was improved using the so-called averaged zero point (AZP) method, ${ }^{23}$ where the helium atoms are described classically but including the zero point motion coming from the definition of a wave function centered at the position of each atom.

The strong correlation found experimentally between the amount of energy released from the molecule to the nanodroplet and the mass of the photofragments in the photodissociation of alkyl iodides ${ }^{18}$ (where a similar energy excess but different masses of the fragments were examined) has encouraged us to carry out the present work. Thus, our main goal here has been to try to understand better the role played by the fragment mass in the photodissociation inside a superfluid helium nanodroplet

$$
\mathrm{X}_{2} \text { (ground state)@ }\left({ }^{4} \mathrm{He}\right)_{N}+h \nu \rightarrow\left[\mathrm{X}_{2}(\text { excited state }) @\left({ }^{4} \mathrm{He}\right)_{N}\right]^{*}
$$

$$
\begin{aligned}
{\left.\left[\mathrm{X}_{2} \text { (excited state }\right) @\left({ }^{4} \mathrm{He}\right)_{N}\right]^{*} \rightarrow } & \mathrm{X}\left({ }^{2} \mathrm{P}_{3 / 2}\right)+\mathrm{X}^{*}\left({ }^{2} \mathrm{P}_{1 / 2}\right)+\left[\left({ }^{4} \mathrm{He}\right)_{N^{\prime}}\right]^{*} \\
& +\left(N-N^{\prime}\right)^{4} \mathrm{He} .
\end{aligned}
$$

Eqn (1a) shows the electronic excitation of the embedded $\mathrm{X}_{2}$ molecule $(\lambda \approx 400 \mathrm{~nm} ; 3.10 \mathrm{eV}){ }^{24}$ eqn $(1 \mathrm{~b})$ refers to the photodissociation process, the $\left(N-N^{\prime}\right)^{4} \mathrm{He}$ term of eqn (1b) only reflects the total number of evaporated He atoms, and here $N$ has been taken to be equal to 300 .

To model this situation in a comprehensive way we have analyzed the photodissociation of several hypothetical $\mathrm{X}_{2}$ isotopic variants of the $\mathrm{Cl}_{2}$ reference system (photodissociation induced by the $\mathrm{B} \leftarrow \mathrm{X}$ electronic transition), ${ }^{25}$ in order to explore in a systematic manner a fairly wide range of masses $\left(m_{\mathrm{X}}: 0.25 m_{\mathrm{Cl}}-1.50 m_{\mathrm{Cl}}\right)$. This means that both the initial ground state vibrational wave function and the potential energies involved in the dynamics have been taken as identical to those of $\mathrm{Cl}_{2}$. Proceeding in this way we can attribute the differences found in the dynamical simulations exclusively to the mass of each specific case analyzed.

This study has been performed using a quantum hybrid approach recently proposed by $\mathrm{us}^{25}$ and previously applied to investigate the dynamics of several physicochemical problems involving $\left({ }^{4} \mathrm{He}\right)_{N}$ and atoms or molecules. ${ }^{25-28}$ Consequently, the helium and the $\mathrm{X}_{2}$ molecule have been described using the time dependent density functional (TDDFT) theory and standard quantum mechanics, respectively, analogously to the $\mathrm{Cl}_{2}$ (B) photodissociation. ${ }^{25,26}$ This method provides a good compromise between the quality of the description of the doped quantum fluid and the computational feasibility of the calculations, and can also be a powerful tool for studying the mechanisms implied in the energy exchange between the helium nanodroplets and the dopant species.

This paper is organized as follows: the theoretical methods used are concisely described in Section 2, the analysis of the most important results obtained is considered in Section 3, and the summary and conclusions are reported in Section 4.

\section{Theoretical method}

The theoretical method used to describe the quantum dynamics of the photodissociation of a homonuclear diatomic molecule, $\mathrm{X}_{2}$, embedded in a superfluid helium nanodroplet has been recently proposed by us. ${ }^{25}$ This method has hybrid character: the helium atoms are described employing the mean field TDDFT method and the $\mathrm{X}_{2}$ molecule is described using a standard time dependent quantum wave packet (WP). Moreover, the energy functional used in the previous reference to describe the helium is the so-called Orsay-Trento (OT) phenomenological functional $(T=0 \mathrm{~K}),{ }^{29}$ with some reasonable and commonly used approximations (the backflow term and the non-local contribution to the helium correlation energy have not been considered). ${ }^{25-28,30-32}$ Therefore, in this section we will only review the main features of the TDDFT/WP approach and address the interested reader to ref. 25 for further details.

Differing from ref. 25 where, as a first application of the TDDFT/WP method, the photodissociation of $\mathrm{Cl}_{2}$ (B excited state) in $\left({ }^{4} \mathrm{He}\right)_{N}$ was investigated analyzing the influence of the nanodroplet size on the dynamics ( $N$ : 50-500), here we have focused the attention on the effect of the atomic mass on the dynamics. To do this in a comprehensive manner we have examined several hypothetical isotopes of the $\mathrm{Cl}_{2}(\mathrm{~B} \leftarrow \mathrm{X}$ electronic transition) reference system, to be able to sample a wide variety of masses for the $\mathrm{X}$ atom $\left(m_{\mathrm{X}}: 0.25 m_{\mathrm{Cl}}, 0.50 m_{\mathrm{Cl}}\right.$, $0.75 m_{\mathrm{Cl}}, 1.00 m_{\mathrm{Cl}}$ and $\left.1.50 m_{\mathrm{Cl}}\right)$.

To consider exclusively the effect of the mass we needed to keep constant the remaining ingredients of the system contributing to the photodissociation process. Hence, the initial $\left(\mathrm{Cl}_{2}\right)$ ground electronic state rovibrational wave function $\varphi_{\mathrm{Cl}_{2}}(v=0, j=0)$, that in the $\mathrm{B} \leftarrow \mathrm{X}$ electronic transition suddenly evolves into the $\mathrm{Cl}_{2} \mathrm{~B}$ potential energy curve, according to the Frank-Condon principle (vertical transition; $c f$. Fig. 1 of ref. 25), and the potential energy curves of $\mathrm{Cl}_{2}(\mathrm{X})$ and $\mathrm{Cl}_{2}(\mathrm{~B})$ have not been modified, when considering the photodissociation of the $\mathrm{X}_{2}$ molecules containing the $\mathrm{X}$ "isotopes".

The choice to maintain the initial rovibrational ground state wave function of $\mathrm{Cl}_{2}(\mathrm{X})$ only produces slight differences in the initial kinetic energy, due to the different masses and the resulting zero point energy (ZPE) considered for the $\mathrm{X}_{2}$ wave function ( $c f$. Table 1). Since this kinetic energy of the $\mathrm{X}_{2}$ molecule in the electronic ground state is related to the uncertainty principle (vibrational motion), rather than to a purely translational kinetic energy, we do not expect this to influence in a significant way the photodissociation process on 
Table 1 Initial time $(t=0)$ energies of the $\mathrm{X}_{2}$ diatomic molecules ${ }^{a}$

\begin{tabular}{llll}
\hline$m_{\mathrm{X}}\left(\mathrm{m}_{\mathrm{Cl}}\right.$ unit $)$ & $\left\langle E_{\text {kin }}\right\rangle(\mathrm{K})$ & $\left\langle E_{\text {tot }}\right\rangle(\mathrm{K})$ & $\left\langle E_{\text {tot }}\right\rangle$ diff. $(\%)$ \\
\hline 0.25 & 797.8 & 11891.9 & 0.0 \\
0.50 & 398.7 & 11492.8 & 3.4 \\
0.75 & 265.8 & 11359.9 & 4.5 \\
1.00 & 199.2 & 11293.3 & 5.0 \\
1.50 & 132.8 & 11226.5 & 5.6
\end{tabular}

${ }^{a}$ Mean values of the zero point energy motion (kinetic energy part only) and total energies. The relative differences between the mean values of $E_{\text {tot }}$ with respect to the $m_{\mathrm{X}}=0.25 m_{\mathrm{Cl}}$ case are also shown.

which we want to focus (the kinetic energy associated with the mean velocity of each $X$ atom a little after the excitation into the B state is much larger than the ZPE). Of course, if we had decided to employ the rovibrational ground state wave function that really corresponds to each mass $\left(\mathrm{X}_{2}\right)$, this would have produced a wide variety of initial $\mathrm{X}_{2}$ potential energies for the dynamics, once the Franck-Condon principle is applied in the $\mathrm{B} \leftarrow \mathrm{X}$ electronic transition. The energies of the $\mathrm{X}_{2}$ model molecules at the initial time $(t=0)$ of the dynamics are collected in Table 1, where it can be seen that the energy differences are in the $3.4-5.6 \%$ interval (taken as a reference the energy of $\mathrm{X}_{2}$ for $\left.m_{\mathrm{X}}=0.25 \mathrm{~m}_{\mathrm{Cl}}\right)$.

A nanodroplet with $300{ }^{4} \mathrm{He}$ atoms has been selected for this study. This nanodroplet size (radius $=17.5 \AA$ ) represents a compromise between the computational cost and dealing with a size comparable to the experiments. Besides, this nanodroplet is large enough to make possible the determination of the two operating modes for the $\mathrm{X}_{2}$-helium energy exchange (cf. Section 3.2). If a smaller nanodroplet had been selected, e.g. the case $N=50$ (and similarly for $N=100$ ), the energy exchange would only result from the collision of the $\mathrm{X}$ fragment atoms with the solvation shell (cavity wall); as after this collision the fragment atoms would essentially be leaving the nanodroplet surface.

In our approach, the evolution of the nanodroplet is coupled with that of the molecule and, due to the symmetry of the problem and assuming a zero angular momentum situation, ${ }^{25}$ for the molecule is only needed to deal with the relative coordinate between the two atoms, which is denoted by $r$. The photodissociation takes place along the molecular axis, which is placed in the $z$-axis, and the origin of coordinates is placed in the centre of the nanodroplet.

The two coupled Schrödinger-like non-linear equations of motion that describe the temporal evolution of the helium effective complex wave function, $\left|\Psi_{\mathrm{He}}\left(\boldsymbol{R}_{\mathrm{He}}, t\right)\right|^{2} \equiv \rho_{\mathrm{He}}\left(\boldsymbol{R}_{\mathrm{He}}, t\right)$, and the relative coordinate molecular wave function (wave packet), $\varphi_{\mathrm{X}_{2}}(r, t)$, read, respectively, as:

$$
\begin{aligned}
\mathrm{i} \hbar \frac{\partial}{\partial t} \Psi_{\mathrm{He}}\left(\boldsymbol{R}_{\mathrm{He}}\right)= & {\left[-\frac{\hbar^{2}}{2 m_{\mathrm{He}}} \nabla^{2}+\int \mathrm{d} r V_{\mathrm{He}-\mathrm{X}_{2}(\mathrm{~B})}\left(r, \boldsymbol{R}_{\mathrm{He}}\right)\left|\varphi_{\mathrm{X}_{2}}(r)\right|^{2}\right.} \\
& \left.+\frac{\delta \mathcal{E}_{\mathrm{c}}\left[\rho_{\mathrm{He}}\right]}{\delta \rho_{\mathrm{He}}}\right] \Psi_{\mathrm{He}}\left(\boldsymbol{R}_{\mathrm{He}}\right)
\end{aligned}
$$

$$
\begin{aligned}
\mathrm{i} \hbar \frac{\partial}{\partial t} \varphi_{\mathrm{X}_{2}}(r)= & {\left[-\frac{\hbar^{2}}{m_{\mathrm{X}}} \frac{\partial^{2}}{\partial r^{2}}+\int \mathrm{d} \boldsymbol{R}_{\mathrm{He}} V_{\mathrm{He}-\mathrm{X}_{2}(\mathrm{~B})}\left(r, \boldsymbol{R}_{\mathrm{He}}\right) \rho_{\mathrm{He}}\left(\boldsymbol{R}_{\mathrm{He}}\right)\right.} \\
& \left.+V_{\mathrm{X}_{2}(\mathrm{~B})}(r)\right] \varphi_{\mathrm{X}_{2}}(r)
\end{aligned}
$$

$\rho_{\mathrm{He}}\left(\mathbf{R}_{\mathrm{He}}\right)$ and $\mathcal{E}_{\mathrm{c}}\left[\rho_{\mathrm{He}}\right]$ are the density and the sum of the correlation and potential energy densities of liquid ${ }^{4} \mathrm{He}$, respectively, the OT functional, that is introduced through the $\frac{\delta \mathcal{E}_{\mathrm{c}}\left[\rho_{\mathrm{He}}\right]}{\delta \rho_{\mathrm{He}}}$ term, describes the superfluid helium, and the $\mathrm{He}-\mathrm{Cl}_{2}(\mathrm{X}, \mathrm{B})$ and $\mathrm{Cl}_{2}(\mathrm{X}, \mathrm{B})$ potential energies have been taken from ref. 33, where the " $\mathrm{X}$ " symbol in the parentheses here refers to the electronic ground state of the $\mathrm{Cl}_{2}$ molecule.

The time propagation of the wave functions has been determined by solving eqn (2a) and (2b) numerically, using discretization techniques and applying standard procedures. Besides, negative imaginary potentials (NIPs) have been added for $\mathrm{X}_{2}$ and helium in order to avoid non-physical reflections of the wave functions at the limits of the grid. ${ }^{25}$

\section{Results and discussion}

\subsection{Photodissociation mechanism}

The aim of this section is to obtain a deep insight into the influence of the atomic mass on the photodissociation dynamics inside superfluid helium nanodroplets. Thus, five different values of the atomic mass have been selected for the $\mathrm{X}_{2}$ diatomic molecule, in order to cover a wide enough mass range keeping the investigation computationally affordable. These masses correspond to $m_{\mathrm{X}}=0.25,0.50,0.75,1.0$ and $1.5 m_{\mathrm{Cl}}$, i.e. expressed in terms of the atomic chlorine $\left({ }^{35} \mathrm{Cl}\right)$ mass. Furthermore, to ensure that the possible differences observed in the dynamical behavior arise exclusively from the mass effect, we have considered the same values for the other initial properties specifying the input data for the dynamics study. Among other things, this framework includes all masses using the same initial relative coordinate wave packet that corresponds to the ground state of the chlorine molecule.

The photodissociation mechanism of $\mathrm{X}_{2}(\mathrm{~B})$ is very similar to that observed for the chlorine molecule in the $\mathrm{B}$ excited electronic state. ${ }^{25,26}$ Therefore, e.g., it takes place in the picosecond timescale with an efficient energy exchange between the molecule and the nanodroplet; and the quantum interference resulting from the interaction of $\mathrm{X}_{2}(\mathrm{~B})$ with the helium environment leads to an oscillating velocity distribution of the atomic (X) photofragments. However, the particularly large energy exchange produced for the lowest masses explored $\left(0.25 m_{\mathrm{Cl}}\right.$ and $0.50 m_{\mathrm{Cl}}$ ) can even induce the geminate recombination of the $\mathrm{X}$ atoms regenerating the $\mathrm{X}_{2}$ molecule. Thus, for $m_{\mathrm{X}}=0.50 m_{\mathrm{Cl}}$ there is a recombination probability of $\approx 86 \%$ while for $m_{\mathrm{X}}=0.25 m_{\mathrm{Cl}}$ the probability is $100 \%$.

In the former case $\left(m_{\mathrm{X}}=0.50 m_{\mathrm{Cl}}\right)$ the wave packet remains for a long time close to the nanodroplet inner surface, until the $\mathrm{X}_{2}$-helium attractive interaction induces the re-solvation 
(at the surface) of a fraction of the $\mathrm{X}_{2}$ wave packet and then it splits into two parts. The solvated fragment recombines and the remaining one leaves the nanodroplet leading to dissociation. The photodissociation process for the $m=0.25 m_{\mathrm{Cl}}$ "isotope" variant has a rather different mechanism, since the energy exchange is even more efficient than that for $m_{\mathrm{X}}=0.50 m_{\mathrm{Cl}}$ and the wave packet comes back to the center of the nanodroplet before reaching the nanodroplet inner surface. The process of recombination, however, does not occur directly, as a "rebound" effect occurs due to the high excitation of the nanodroplet that produces density waves.

Some snapshots showing the time evolution of the $\mathrm{X}_{2}$ wave packet probability density and the effective potential energy that governs its dynamics (i.e. the sum of the $\mathrm{Cl}_{2}(\mathrm{~B})$ potential energy and the $\mathrm{Cl}_{2}(\mathrm{~B})$-helium interaction potential energy) ${ }^{25}$ are shown in Fig. 1a for the case $m_{\mathrm{X}}=0.25 m_{\mathrm{Cl}}$, in order to visualize its unsuccessful photodissociation mechanism. Important oscillations are formed in the wave packet due to the collision with the first solvation shell, which are progressively attenuated, and the confinement provided by the surrounding helium avoids the continuous spatial spreading of the wave packet with time.

The evolution of the helium density ( $x z$ plane) is presented in Fig. $1 \mathrm{~b}$ and $\mathrm{c}$ (three- and two-dimensional representations, respectively). At the beginning of the process strong distortions are generated in the helium density, mainly in the vicinity of the $z$ axis (photodissociation axis), and shock waves are produced due to the collision of the wave packet with the first solvation shell. Once the shock waves collide with the nanodroplet surface some helium density escapes from the nanodroplet (evaporation process). The circular like black areas observed inside the nanodroplet in the two-dimensional plots (Fig. 1c) correspond to the cavities formed around the $\mathrm{X}_{2}$ molecule or the $\mathrm{X}$ atoms (depending on the time values considered) where no helium can be found.

It is worth noting that the geminate recombination is induced by the geometry, i.e. by the fact that we are dealing with nanodroplets (finite systems) rather than by the nature of the liquid. Before the lightest atoms $\left(m_{\mathrm{X}}=0.25 m_{\mathrm{Cl}}\right)$ reach the inner surface of the nanodroplet, the $\mathrm{X}$-helium net attractive interaction force acting on them arising from the asymmetric helium distribution around each atom (one sided forces in the vicinity of the surface) is able to stop them and change the direction of their velocities. Then, both $\mathrm{X}$ atoms start moving towards the center of the nanodroplet, progressively approaching each other, and, finally, the recombination takes place. However, if the velocity of the $\mathrm{X}$ atoms is high enough ( $m_{\mathrm{X}}$ above $0.50 m_{\mathrm{Cl}}$, approximately) the force generated by the asymmetric distribution of helium is not capable of stopping them and, consequently, they are able to leave the nanodroplet completing the photodissociation process. Indeed, it should be mentioned that if the photodissociation would take place in superfluid bulk liquid helium, there would not be a driving net force coming from the impurity-helium interaction motivating the approach of the two $\mathrm{X}$ atoms.

The temporal evolution of the mean value of the relative coordinate and its corresponding velocity are shown in Fig. 2 and 3, respectively. These plots end when the NIP begins to absorb the wave packet, at a large distance from the nanodroplet. The general behaviour is coincident with that observed in the case of the $\mathrm{Cl}_{2}$ photodissociation; ${ }^{25}$ but as for the $\mathrm{X}_{2}$ molecules with the lower masses $\left(m_{\mathrm{X}}=0.25 m_{\mathrm{Cl}}\right.$ and $\left.0.50 m_{\mathrm{Cl}}\right)$ the energy exchange is even more effective than that for $\mathrm{Cl}_{2}$, the atomic $\mathrm{X}$ fragments can be stopped leading to the geminate recombination process (i.e. negative relative velocities can occur; $c f$. the inner panel of Fig. 3 ). In Fig. 2 and 3 the "rebound" effect taking place during the $\mathrm{X}+\mathrm{X}^{*}$ recombination can also be observed for the $m_{\mathrm{X}}=0.25 m_{\mathrm{Cl}}$ case.

The most important general feature observed is the strong relationship that exists between the mass of the atoms and the magnitude of energy exchange between the dopant molecule and the helium: the lower the mass, the higher the energy exchange; and in the photodissociation process this may even lead to the recombination of the atomic fragments inside the nanodroplet, as we have already mentioned (for $m_{\mathrm{X}}=0.25 m_{\mathrm{Cl}}$ the whole wave packet recombines, while for $m_{\mathrm{X}}=0.50 m_{\mathrm{Cl}}$ only a small fraction of the wave packet $(\approx 14 \%)$ leaves the nanodroplet). In Table 2 are given the final mean values of the (kinetic) energy and velocity of the atomic photofragments. The final mean energy increases with the atomic mass and varies from 7.0 to $29.3 \%$ of the initial total mean energy of $\mathrm{X}_{2}$ within the $m_{\mathrm{X}}: 0.75 m_{\mathrm{Cl}}-1.50 m_{\mathrm{Cl}}$ interval.

To conclude this section, we analyze the velocity distribution of the leaving $\mathrm{X}$ atoms ( $c f$. Fig. 4 ), which is the main observable that can be measured in the $\mathrm{X}_{2}$ doped nanodroplet. This distribution is strongly oscillating for all the masses leading to photodissociation products, as it was already observed in the case of $\mathrm{Cl}_{2}\left(m_{\mathrm{X}}=1.00 m_{\mathrm{Cl}}\right){ }^{25,26}$ These oscillations arise from quantum interference resulting from the interaction of $\mathrm{X}_{2}(\mathrm{~B})$ with the ${ }^{4} \mathrm{He}$ environment in the early times of the photodissociation (0.00-0.20 ps; $c f$. ref. 26). Besides, from the velocity distribution of $m_{\mathrm{X}}=0.50 m_{\mathrm{Cl}}$ it can be seen that the small lower limit value of the velocity occurs at around $50 \mathrm{~m} \mathrm{~s}^{-1}$.

\subsection{Energy exchange mechanism}

In order to obtain deeper insights into the mechanism of the $\mathrm{X}_{2}$-helium energy exchange, the temporal evolution of the $\mathrm{X}_{2}$ molecule energy (kinetic $+\mathrm{X}-\mathrm{X}$ potential energies) and the energy per helium atom of the nanodroplet has been plotted in Fig. 5a and b, respectively. From these figures two markedly different dynamic regimes are evident: at the very initial times, from $t=0$ to around $0.10-0.15 \mathrm{ps}$ (depending on the case), and the rest of the time, from around $0.10-0.15 \mathrm{ps}$ to the end of the photodissociation process. From Fig. $5 \mathrm{a}$ and $\mathrm{b}$ it is clear that the fastest energy exchange is produced at the very initial times.

The first dynamic regime corresponds to the initial collision of the $\mathrm{X}$ atoms with the walls of the helium cavity (i.e. the volume found inside the nanodroplet and centered in the $\mathrm{X}_{2}$ molecule where no He atoms are found; a cavity radius of about $4.8 \AA$ ). ${ }^{25}$ It should be noted that in a very short time period after the $\mathrm{B} \leftarrow \mathrm{X}$ electronic transition (from $t=0$ to $\approx 0.01 \mathrm{ps}$ ) the initial potential energy of the molecule in the excited state is transformed into kinetic energy of $\mathrm{X}_{2}(\mathrm{~B})$, due to the shape of the B potential energy curve. 


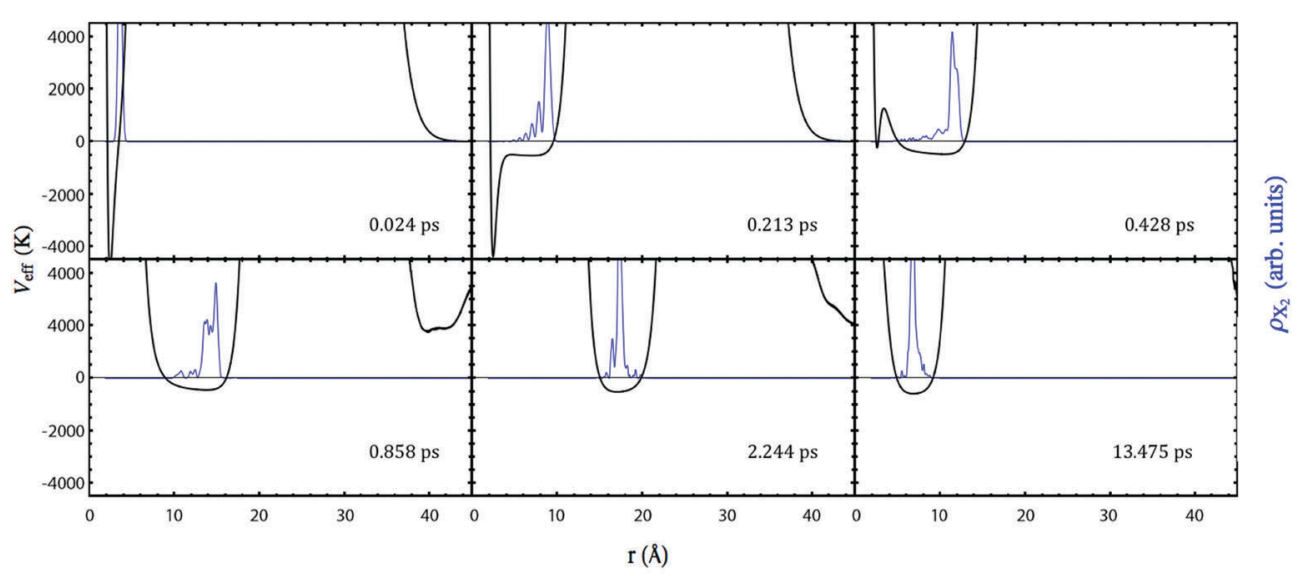

b

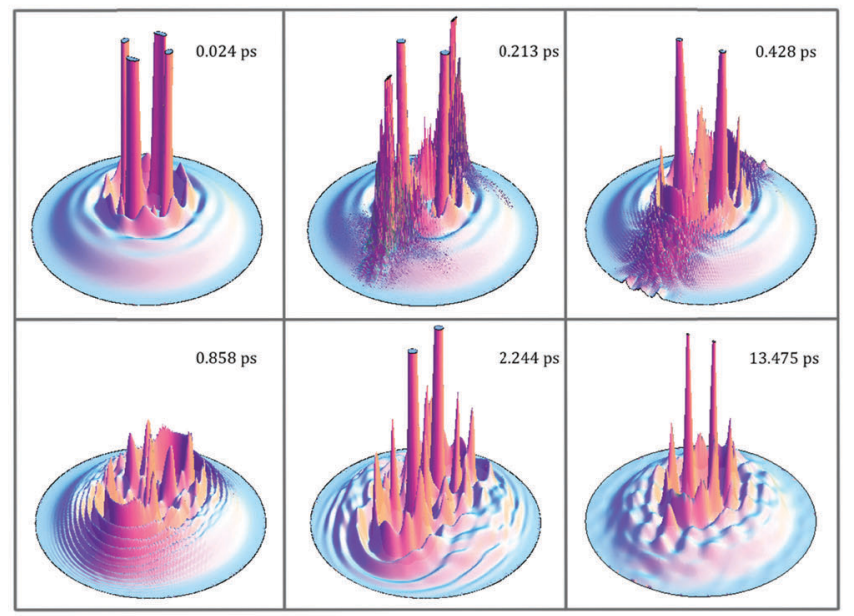

$\mathrm{c}$

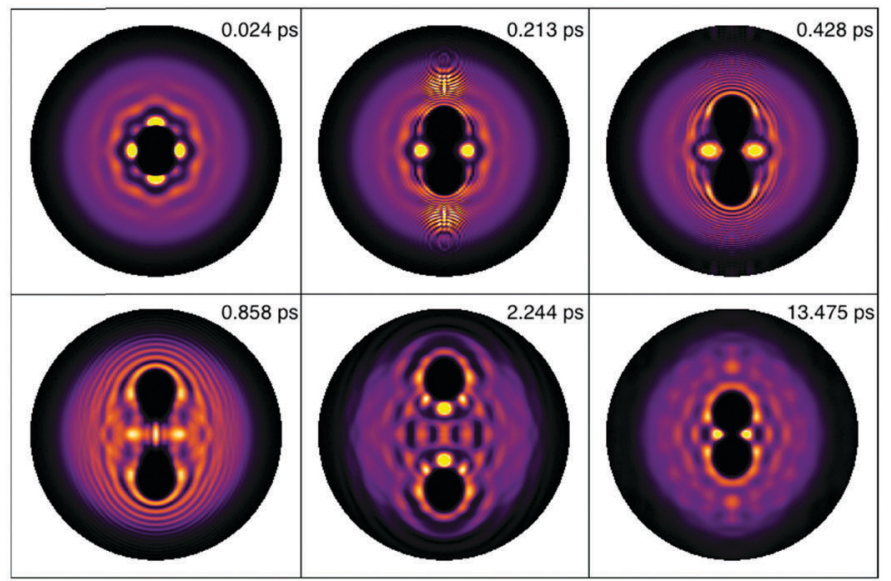

Fig. 1 Snapshots of the $X_{2}\left(m_{X}=0.25 m_{C l}\right)$ squared modulus of the relative coordinate wave packet (blue) and the effective potential energy (black) (a); snapshots of the helium density in the $x z$-plane (three- (b) and two-dimensional (c) representations) at the same time values as in (a). The $z$-axis corresponds to the molecular photodissociation axis and in (c) the highest helium density is indicated in yellow, while the absence of helium is indicated in black.

The temporal evolution of the $\mathrm{X}_{2}$-helium interaction potential energy, $\left\langle E_{\text {int }}\right\rangle$, is given in Fig. 6 . The $\left\langle E_{\text {int }}\right\rangle$ value for the initial and main peak (repulsive interaction energy), which corresponds to the initial collision between the $\mathrm{X}$ atoms and the walls of the cavity, increases as the $\mathrm{X}$ mass decreases. This indicates that for the lighter masses the walls of the helium 


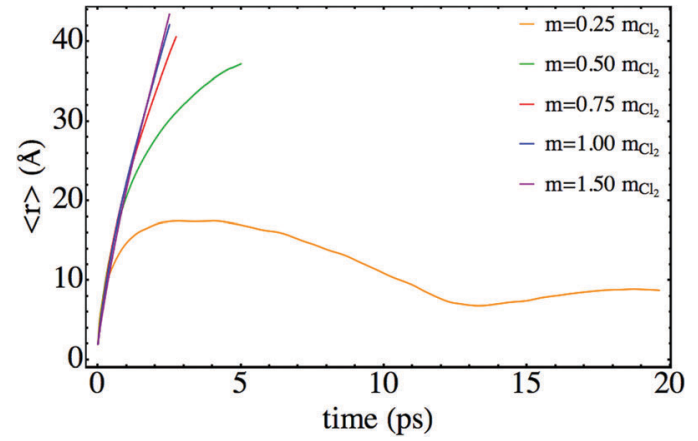

Fig. 2 Time evolution of the mean value of the relative coordinate.

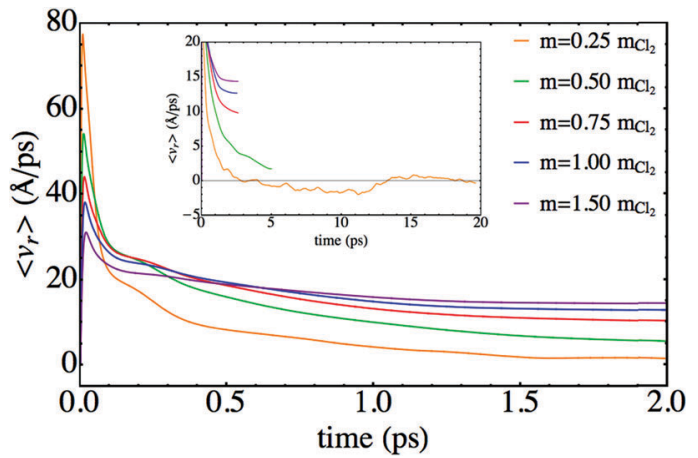

Fig. 3 Time evolution of the mean value of the relative velocity. The inset shows the time evolution for the two lower masses at higher time values.

Table 2 Mean values of the $X+X^{*}$ (kinetic) energy and of the atomic fragments velocity distributions at the end of the photodissociation process

\begin{tabular}{llll}
\hline$m_{\mathrm{X}}\left(m_{\mathrm{Cl}}\right.$ unit $)$ & $\left\langle E_{\text {tot }}\right\rangle$ final $(\mathrm{K})$ & $\%$ of $\left\langle E_{\text {tot }}\right\rangle$ initial & $\left\langle v_{\mathrm{X}}\right\rangle$ final $\left(\mathrm{m} \mathrm{s}^{-1}\right)$ \\
\hline 0.25 & - & - & - \\
0.50 & - & - & $242^{a}$ \\
0.75 & 790.8 & 7.0 & 493 \\
1.00 & 1736.5 & 15.4 & 628 \\
1.50 & 3294.2 & 29.3 & 716
\end{tabular}

${ }^{a}$ This value only corresponds to the outgoing part of the $\mathrm{X}_{2}$ relative motion wave function.

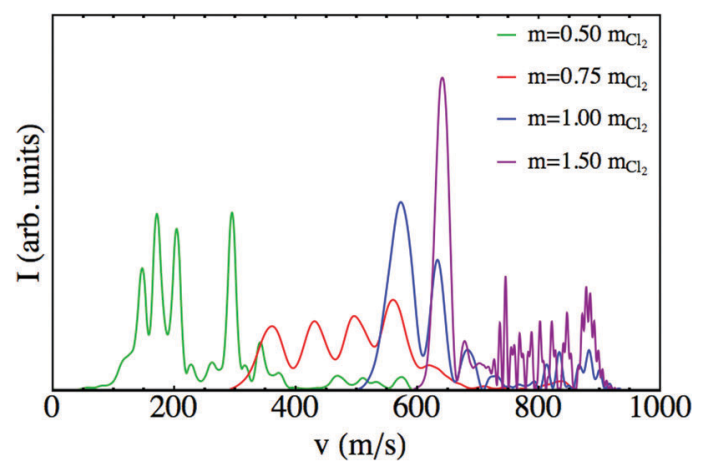

Fig. 4 Velocity distribution of the atomic $(\mathrm{X})$ photofragments.

cavity and the $\mathrm{X}$ atoms get closer to each other than for the larger masses, due to the higher velocities reached by the lower masses, which lead to a collision of more sudden character.

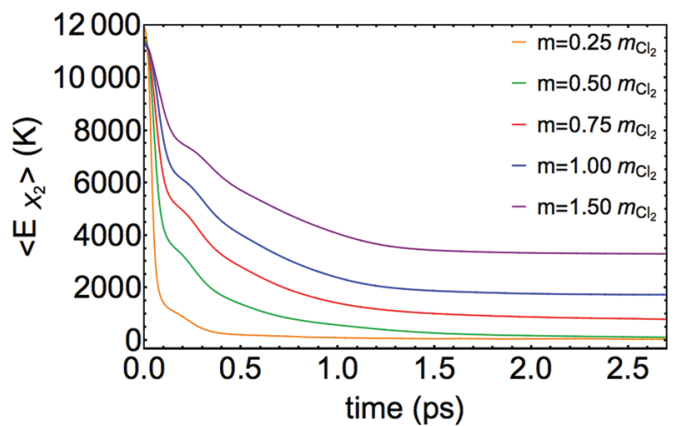

$\mathbf{a}$

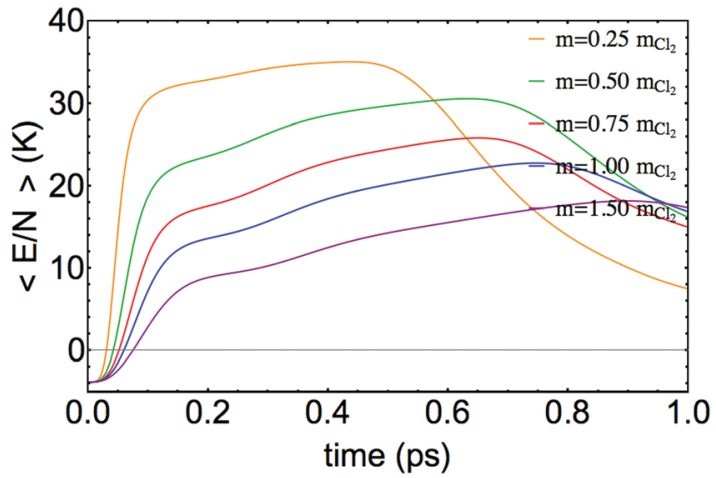

Fig. 5 Time evolution of the mean value of the $X_{2}$ energy (kinetic $+X_{2}$ potential energies) (a) and time evolution of the mean value of the energy per helium atom of the nanodroplet (the decrease of this magnitude results from the evaporation process) (b).

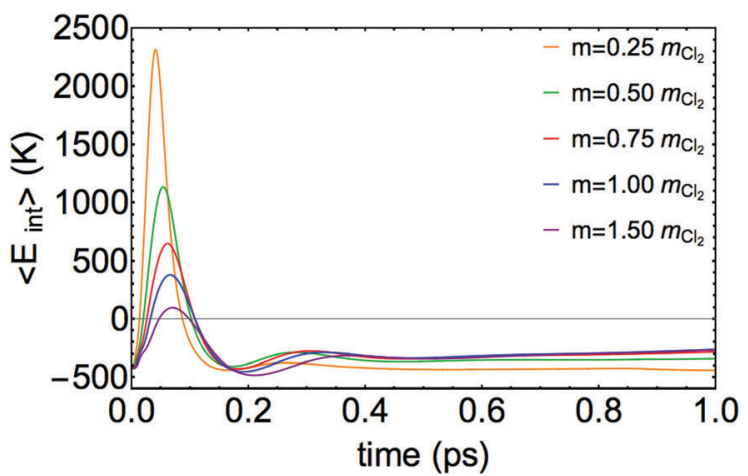

Fig. 6 Time evolution of the mean value of the $X_{2}$-helium interaction potential energy.

However, at the end of this collision the $\mathrm{X}_{2}$-helium interaction potential energy decreases in an important way and becomes negative (of the order of a few hundreds of Kelvin; $c f$. Fig. 6 and Table 3), with the only exception of what happens for the lowest mass. This means that the $\mathrm{X}$ atoms and the surrounding liquid helium have been adapted to each other in a substantial manner. Because of this at the end of the first dynamic regime both the $\mathrm{X}$ atoms and the helium atoms placed in the cavity walls are moving at a rather similar velocity. Thus, the $\mathrm{X}$ atoms, which were initially moving very fast, push the surrounding helium atoms, which were initially at rest, until they reach an essentially coincident velocity. 
Table 3 Frontier time between the two $X_{2}$-helium energy exchange regimes and mean values of the total energy of $X_{2}$ (kinetic + potential energies) and $\mathrm{X}_{2}$-helium interaction energy at this time ${ }^{a}$

\begin{tabular}{llll}
\hline$m_{\mathrm{X}}\left(m_{\mathrm{Cl}}\right.$ unit $)$ & Frontier time $(\mathrm{ps})$ & $\left\langle E_{\text {tot }}\right\rangle$ frontier $(\mathrm{K})$ & $\left\langle E_{\text {int }}\right\rangle$ frontier $(\mathrm{K})$ \\
\hline 0.25 & 0.078 & 1864 & 228 \\
0.50 & 0.120 & 3900 & -229 \\
0.75 & 0.128 & 5619 & -243 \\
1.00 & 0.138 & 6545 & -293 \\
1.50 & 0.159 & 7717 & -379
\end{tabular}

${ }^{a}$ The initial total energy of $\mathrm{X}_{2}$ is in the range of $11892-11227 \mathrm{~K}$ (cf. Table 1).

These results serve as inspiration to formulate a model to account for the initial dynamic regime. This model is based on an initial perfectly inelastic collision between an $\mathrm{X}$ atom of mass $m_{\mathrm{X}}$, moving originally at the velocity $v_{\mathrm{i}}$ and an "effective" particle (helium atoms involved) of mass $M$ originally at rest (a more detailed explanation is given after eqn (3)). At the end of this collision both species are moving together at the velocity $v_{\mathrm{f}}$. Moreover, as the two $\mathrm{X}$ atoms are well separated when the collision is produced their interaction potential energy can be neglected and we can consider only their kinetic energy $\left(E_{\mathrm{i}, \mathrm{f}}\right)$, which would be half of the kinetic energy corresponding to the reduced mass associated with the $\mathrm{X}-\mathrm{X}$ relative motion. Thus, the velocities of each one of the $\mathrm{X}$ atoms before and after the collision $\left(v_{\mathrm{i}, \mathrm{f}}\right)$ are given by $v_{\mathrm{i}, \mathrm{f}}=\sqrt{2 E_{\mathrm{i}, \mathrm{f}} / m_{\mathrm{X}}}$ and imposing the conservation of the total linear momentum $\left(m_{\mathrm{X}} v_{\mathrm{i}}=\left(m_{\mathrm{X}}+M\right) v_{\mathrm{f}}\right)$ we arrive at a rather simple relation between the atomic (X) kinetic energy ratio and the atomic mass $m_{\mathrm{X}}$ :

$$
\left(\sqrt{\frac{E_{\mathrm{i}}}{E_{\mathrm{f}}}}-1\right)^{-1}=m_{\mathrm{X}} / M
$$

In this classical mechanics model $E_{\mathrm{i}}$ corresponds to the kinetic energy attained by each one of the $\mathrm{X}$ atoms once the initial potential energy resulting from the $\mathrm{B} \leftarrow \mathrm{X}$ vertical excitation (energy taken with respect to the $\mathrm{X}+\mathrm{X}^{*}$ dissociated atoms) has been transformed into kinetic energy. This conversion from potential to kinetic energy occurs in a very short time $(\approx 0.01 \mathrm{ps})$ and well before the collision of the $\mathrm{X}$ atoms with the surrounding helium atoms has taken place. Moreover, $E_{\mathrm{f}}$ corresponds to the kinetic energy of each one of the $\mathrm{X}$ atoms once the perfectly inelastic collision with the surrounding helium atoms has occurred, i.e. once the $\mathrm{X}$ atoms have reached the wall of the cavity, the radius of which $(\approx 4.8 \AA)^{25}$ is about two times the equilibrium distance of $\mathrm{Cl}_{2}(\mathrm{~B}) \cdot{ }^{25}$ The time required for this collision to occur depends on the mass of the atoms and is in the $0.08-0.16 \mathrm{ps}$ time interval ( $c f$. Table 3).

Plotting the left side of eqn (3) as a function of $m_{\mathrm{X}}$ we obtain a remarkable good agreement with the linear behavior predicted by this equation (Fig. 7). Hence, from the energy exchange perspective, the initial step for the photodissociation of a homonuclear diatomic molecule inside a helium nanodroplet can be rationalized in terms of a perfectly inelastic collision of the $\mathrm{X}$ atoms with the cavity wall. From the analysis of the data represented in Fig. 7 we determine a value of $M$

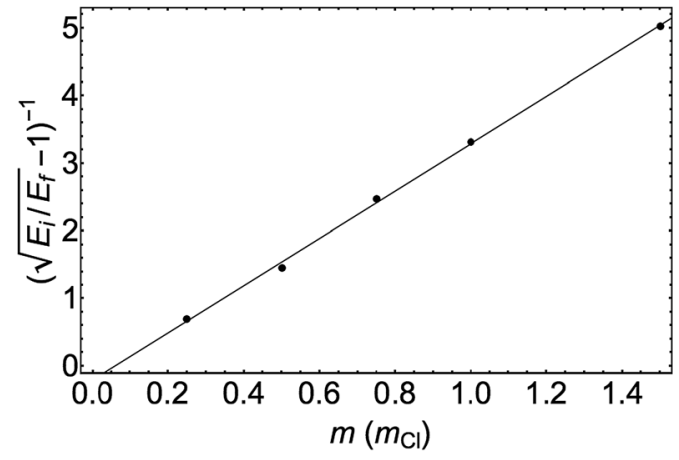

Fig. 7 Dependence of the $\left(\sqrt{\frac{E_{\mathrm{i}}}{E_{\mathrm{f}}}}-1\right)^{-1}$ expression as a function of the atomic mass $\left(m_{\mathrm{X}}\right)$, for the first dynamic regime (perfectly inelastic collision) of the photodissociation process.

equal to 10.1 amu; which means that in the early step of the photodissociation process each $\mathrm{X}$ atom collides and pushes on average a little more than two He atoms.

In order to discern whether the perfectly inelastic collision involved in this part of the process arises from the presence of snowball structures in the initial helium configuration, we have carried out an analogous calculation, but using a hypothetic initial helium density without snowballs, and the same pattern has been found. Therefore, the reproduction of the theoretical results on the basis of a perfectly inelastic collision results from the induction of movement in helium as a result of the collision and not from the collision with a rigid snowball structure. Furthermore, it is worth noting that the nanodroplet induced geminate recombination of $\mathrm{X}+\mathrm{X}^{*}$ to lead to $\mathrm{X}_{2}(\mathrm{~B})$ for the lower masses is mainly caused by the first part of the photodissociation process, because the $\mathrm{X}_{2}$-helium energy exchange is particularly large.

The second dynamic regime for the energy exchange refers to the situation in which the atoms are travelling through the nanodroplet at velocities higher than the Landau critical velocity. In the previous investigation on the $\mathrm{Cl}_{2}$ photodissociation, ${ }^{25}$ from the $\left\langle v_{r}\right\rangle v s$. $\langle r\rangle$ representation, we pointed out that a viscous fluid model could be applied in this context, but without analyzing it in quantitative terms.

Here, the exploration of different masses $\left(m_{\mathrm{X}}\right)$ allowed us to examine this question in a detailed way, reinforcing the previous qualitative arguments ${ }^{25}$ by comparison of the friction parameters $(b)$ obtained in the different simulations. A velocity of each $\mathrm{X}$ atom proportional to the friction force, $F=m_{\mathrm{X}} \frac{\mathrm{d} v}{\mathrm{~d} t}=-b v$, leads to a linear relationship between the velocity $(v)$ and the distance travelled by the $\mathrm{X}$ atom $(z)$ :

$$
v(z)=v_{\mathrm{f}}-\frac{b}{m_{\mathrm{X}}} z
$$

The dependence of $\left\langle v_{r}\right\rangle$ versus $\langle r\rangle$ for the second regime of the energy exchange presents a rather linear shape in all the cases (Fig. 8). Defining $v(z) \equiv\left\langle v_{r}\right\rangle / 2$ and $z \equiv\langle r\rangle / 2$ similar values of the friction parameter $b$ have been found for all the masses (Table 4). The resulting mean value is $b=0.37$ atomic units. 


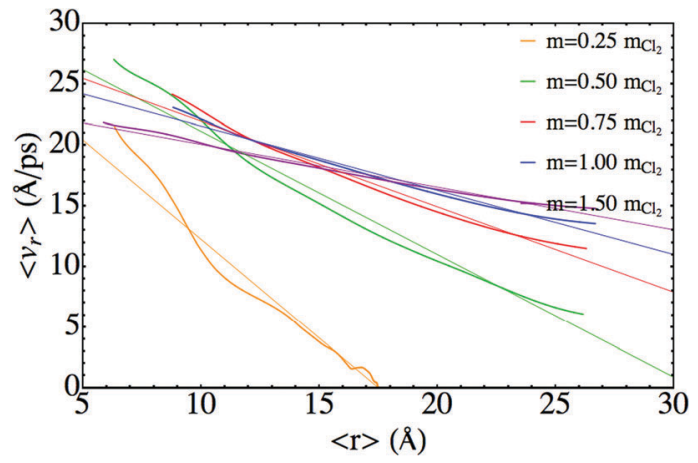

Fig. 8 Dependence of $\left\langle v_{r}\right\rangle$ as a function of $\langle r\rangle$, for the second dynamic regime (viscous flow) of the photodissociation process.

Table 4 Friction coefficients

\begin{tabular}{ll}
\hline$m_{\mathrm{X}}\left(m_{\mathrm{Cl}}\right.$ unit $)$ & $b(\mathrm{au})$ \\
\hline 0.25 & 0.32 \\
0.50 & 0.40 \\
0.75 & 0.41 \\
1.00 & 0.41 \\
1.50 & 0.39
\end{tabular}

This value is about five times the friction coefficient obtained experimentally from the mobility of $\mathrm{Na}^{+}$in superfluid bulk liquid helium at $T=1.42 \mathrm{~K}(b=0.078 \mathrm{au}) .{ }^{34}$ Moreover, in a theoretical study of the fragmentation of $\mathrm{Ne}_{3}{ }^{+}$in helium nanodroplets, where the helium environment was modelled by a friction force, from the comparison with the experimental data a lower $(0.025 \mathrm{au})$ and a upper $(1.5 \mathrm{au})$ limit were proposed for the friction coefficient of $\mathrm{Ne}^{+} .22$

In addition, we have recently published a dynamics investigation on the capture of a Ne atom by a helium nanodroplet using the same theoretical framework employed here. ${ }^{27}$ When the mean value of the velocity is represented versus the mean value of position we also found a linear dependence for the $\mathrm{Ne}$ atom velocities above the Landau critical velocity. From the plots in Fig. 4 of ref. 13 a value of $b$ equal to 0.38 au is obtained, which is very close to the values obtained from the present photodissociation study.

It is worth noting here that, for the lower mass considered $\left(m_{\mathrm{X}}=0.25 m_{\mathrm{Cl}}\right)$, in the recombination process the $\mathrm{X}$ atoms come back towards the nanodroplet center at rather low absolute values of the mean velocity ( $c f$. Fig. 3). Moreover, even though at some time values the velocity of each $\mathrm{X}$ atom is below the Landau critical velocity $\left(\approx 58 \mathrm{~m} \mathrm{~s}^{-1}\left(0.58 \AA \mathrm{ps}^{-1}\right)\right.$ in superfluid (bulk) helium ${ }^{35,36}$ and, due to the reasonable simplifications made in the OT functional to make possible this type of study, the theoretical value is $\approx 90 \mathrm{~m} \mathrm{~s}^{-131}$ ), it does not remain constant (in absolute value).

This is caused by the highly excited helium density waves which are present in the nanodroplet. When these waves collide with the atoms some energy and momentum are exchanged between them and due to this the velocity of the $\mathrm{X}$ atoms is not constant over time. This is in contrast to the behavior found in the capture of a Ne atom, as once the atom mean velocity is below the critical velocity it remains constant in absolute value (cf. Fig. 3 and 4 of ref. 27). The energy conditions examined in the Ne capture are much softer $\left(\left\langle E_{\mathrm{kin}}\right\rangle \text { initial: 9.74-2031.7 K }\right)^{27}$ than those in the present photodissociation study and this is the main reason for the observed differences.

To conclude the present analysis we have estimated the viscosity $(\eta)$ of liquid helium from the values of the friction force parameter $(b)$ and the van der Waals radius of the $\mathrm{X}$ atoms (i.e. the $\mathrm{Cl}$ value), assuming a Stokes flow of a sphere. We have obtained a value of $\eta=4.41 \times 10^{-6} \mathrm{~Pa}$ s from the $\mathrm{X}_{2}$ data obtained here and $\eta=4.95 \times 10^{-6} \mathrm{~Pa}$ s from the Ne data of ref. 27. These similar values are comparable with the experimental results for superfluid bulk liquid helium at saturated vapor pressure $\left(\right.$ e.g. $\eta=2.385 \times 10^{-6}, 3.028 \times 10^{-6}, 4.806 \times 10^{-6}$ and $9.537 \times 10^{-6} \mathrm{~Pa} \mathrm{~s}$ for $T=2.17,1.05,0.95$ and $0.85 \mathrm{~K}$, respectively). ${ }^{37}$

\section{Summary and conclusions}

In this work we have carried out a theoretical study on the influence of the mass in the photodissociation dynamics of a homonuclear diatomic molecule $\left(\mathrm{X}_{2}\right)$ embedded in a superfluid helium nanodroplet $(T=0.37 \mathrm{~K}$ ) of 300 atoms ( $c f$. eqn (1)). To do this we have employed a hybrid quantum dynamics method recently proposed by us (TDDFT (helium) + time dependent WP $\left(\mathrm{X}_{2}\right)$ ) and considered several hypothetical isotopes of the $\mathrm{Cl}_{2}$ reference system ( $\mathrm{B} \leftarrow \mathrm{X}$ electronic transition) in order to sample a wide variety of masses $\left(m_{\mathrm{X}}: 0.25 m_{\mathrm{Cl}}-1.50 m_{\mathrm{Cl}}\right)$. To the best of our knowledge this is the first time that this problem has been investigated theoretically.

The photodissociation mechanism of $\mathrm{X}_{2}(\mathrm{~B})$ is very similar to that of $\mathrm{Cl}_{2}(\mathrm{~B})$. It occurs in the picosecond timescale, there is an efficient $\mathrm{X}_{2}$-helium energy exchange and the existence of quantum interference results in an oscillating velocity distribution of the dissociating $\mathrm{X}$ atoms.

The efficiency of the $\mathrm{X}_{2}$-helium energy exchange mechanism increases in a substantial way when the $\mathbf{X}$ atom mass $\left(m_{\mathrm{X}}\right)$ decreases. Thus, the energy exchanged from the molecule to the nanodroplet can be so large that it leads to the full and partial $(\approx 86 \%)$ geminate recombination of the dissociating $\mathrm{X}$ atoms, for the lower mass values investigated here $\left(m_{\mathrm{X}}=0.25 m_{\mathrm{Cl}}\right.$ and $m_{\mathrm{X}}=0.50 m_{\mathrm{Cl}}$, respectively).

A detailed analysis of the photodissociation process has shown that, from the point of view of the energy exchange, it consists of two main steps. The former step occurs at the initial times of the photodissociation and can be rationalized in terms of a perfectly inelastic collision occurring between the atomic photofragments $(\mathrm{X})$ and some helium atoms of the solvation shell. In the latter step the atomic photofragments are moving through the nanodroplet that behaves as a viscous fluid.

The two dynamic regimes for the energy exchange found in the present study (perfectly inelastic collision (IC) + viscous flow (VF); ICVF mechanism) are probably of general character 
for the photodissociation of molecules embedded in superfluid helium nanodroplets.

\section{Acknowledgements}

This work has been supported by the Spanish Ministry of Science and Innovation (project ref. CTQ2011-27857-C02-01), and we also want to acknowledge the support from the Autonomous Government of Catalonia (A. V. predoctoral fellowship and project ref. 2009SGR 17, 2014SGR 25 and XRQTC). Thanks are also given to Professor Ricardo Mayol (University of Barcelona) for helpful comments on the theoretical description of helium and reading the manuscript.

\section{References}

1 J. P. Toennies and A. Vilesov, Angew. Chem., Int. Ed., 2004, 43, 2622.

2 M. Barranco, R. Guardiola, S. Hernández, R. Mayol, J. Navarro and M. Pi, J. Low Temp. Phys., 2006, 142, 1.

3 A. Slenczka and J. P. Toennies, in Low Temperature and Cold Molecules, ed. I. W. M. Smith, Imperial College Press, London, 2008, pp. 345-392.

4 S. Yang and A. M. Ellis, Chem. Soc. Rev., 2013, 42, 472.

5 J. Tiggesbäumker and F. Stienkemeier, Phys. Chem. Chem. Phys., 2007, 9, 4748.

6 S. Yang, A. M. Ellis, D. Spence, C. Feng, A. Boatwright, E. Latimer and C. Binns, Nanoscale, 2013, 5, 11545.

7 L. F. Gomez, E. Loginov and A. F. Vilesov, Phys. Rev. Lett., 2012, 108, 155302.

8 E. Latimer, D. Spence, C. Feng, A. Boatwright, A. M. Ellis and S. Yang, Nano Lett., 2014, 14, 2902.

9 A. Scheidemann, B. Schilling and J. P. Toennies, J. Phys. Chem., 1993, 97, 2128.

10 B. E. Callicoatt, D. D. Mar, V. A. Apkarian and K. C. Janda, J. Chem. Phys., 1996, 105, 7872.

11 W. K. Lewis, B. E. Applegate, J. Sztáray, B. Sztáray, T. Baer, R. J. Bemish and R. E. Miller, J. Am. Chem. Soc., 2004, 126, 11283.

12 W. K. Lewis, R. J. Bemish and R. E. Miller, J. Chem. Phys., 2005, 123, 141103.

13 B. E. Callicoatt, K. Fröde, L. F. Jung, T. Ruchti and K. C. Janda, J. Chem. Phys., 1998, 109, 10195.

14 B. E. Callicoatt, K. Fröde, T. Ruchti, L. Jung, K. C. Janda and N. Halberstadt, J. Chem. Phys., 1998, 108, 9371.

15 T. Ruchti, K. Fröde, B. E. Callicoatt, H. Ludwigs and K. C. Janda, J. Chem. Phys., 1998, 109, 10679.
16 T. Ruchti, B. E. Callicoatt and K. C. Janda, Phys. Chem. Chem. Phys., 2000, 2, 4075.

17 G. E. Douberly, J. M. Merritt and R. E. Miller, Phys. Chem. Chem. Phys., 2005, 7, 363.

18 A. Braun and M. Drabbels, J. Chem. Phys., 2007, 127, 114303.

19 A. Braun and M. Drabbels, J. Chem. Phys., 2007, 127, 114304.

20 A. Braun and M. Drabbels, J. Chem. Phys., 2007, 127, 114305.

21 T. Takayanagi and M. Shiga, Chem. Phys. Lett., 2003, 372, 90.

22 D. Bonhommeau, A. Viel and N. Halberstadt, J. Chem. Phys., 2004, 120, 11359.

23 D. Bonhommeau, M. Lewerez and N. Halberstadt, J. Chem. Phys., 2008, 128, 054302.

24 Y. Asano and S. Yabushita, J. Phys. Chem. A, 2001, 105, 9873.

25 A. Vilà, M. González and R. Mayol, J. Chem. Theory Comput., 2015, 11, 899.

26 A. Vilà, M. González and R. Mayol, Phys. Chem. Chem. Phys., 2015, 17, 32241.

27 A. Vilà, M. González and R. Mayol, Phys. Chem. Chem. Phys., 2016, 18, 2006.

28 A. Vilà, M. González and R. Mayol, Phys. Chem. Chem. Phys., 2016, 18, 2409.

29 F. Dalfovo, A. Lastri, L. Pricaupenko, S. Stringari and J. Treiner, Phys. Rev. B: Condens. Matter Mater. Phys., 1995, 52, 1193.

30 D. Mateo, A. Hernando, M. Barranco, E. Loginov, M. Drabbels and M. Pi, Phys. Chem. Chem. Phys., 2013, 15, 18388.

31 N. B. Brauer, S. Smolarek, E. Loginov, D. Mateo, A. Hernando, M. Pi, M. Barranco, W. J. Bruma and M. Drabbels, Phys. Rev. Lett., 2013, 111, 153002.

32 D. Mateo, F. Gonzalez and J. Eloranta, J. Phys. Chem. A, 2015, 119, 2262.

33 J. Williams, A. Rohrbacher, J. Seong, N. Marianayagam, K. C. Janda, R. Burcl, M. M. Szczezśniak, G. Chałasiński, S. M. Cybulski and N. Halberstadt, J. Chem. Phys., 1999, 111, 997.

34 H. Günther, M. Foerste, G. zu Putlitz and T. Schumacher, Low Temp. Phys., 1996, 22, 143.

35 D. R. Tilley and J. Tilley, Condensates and Excitations. Superfluidity and Superconductivity, Institut of Physics Publishing, Bristol, 1990, pp. 41-53.

36 J. F. Annet, Superfluid Helium-4. Superconductivity, Superfluids and Condensates, Oxford University Press, Oxford, 2004, pp. 38-43.

37 R. J. Donnelly and C. F. Barenghi, J. Phys. Chem. Ref. Data, 1998, 27, 1217. 